\title{
Mapping between the dynamic and mechanical properties of the relativistic oscillator and Euler free rigid body
}

\author{
Alberto MOLGADO ${ }^{a}$ and Adán RODRÍGUEZ ${ }^{b}$ \\ ${ }^{a}$ Facultad de Ciencias, Universidad de Colima \\ Bernal Díaz del Castillo 340, Villas San Sebastián \\ 28045 Colima, México \\ E-mail: albertom@ucol.mx \\ ${ }^{b}$ Instituto de Física, Universidad Autónoma de San Luis Potosí \\ Av. Manuel Nava 6, Zona Universitaria, \\ 78290 San Luis Potosí, SLP, México \\ E-mail: adnrdz@ifisica.uaslp.mx
}

Received March 3, 2007; Accepted in Revised Form June 12, 2007

\begin{abstract}
In this work we investigate a formal mapping between the dynamical properties of the unidimensional relativistic oscillator and the asymmetrical rigid top at a classical level. We study the relativistic oscillator within Yamaleev's interpretation of Nambu mechanics. Such interpretation is based on the factorisation of the momenta, and as a consequence of this factorisation we are led to a three dimensional phase space. Solutions of the relativistic oscillator are given in terms of the Jacobian elliptic functions and hence we establish a correspondence of these solutions in terms of well known quantities from the rigid body theory. We also study some mechanical restrictions that appear in the mathematical development of the mapping. In particular, we find a lower bound for the relativistic frequency in order to make the mapping self-consistent and physically legitimate.
\end{abstract}

\section{Introduction}

Historically, Nambu three order phase space formalism of mechanics was developed as a mathematical tool expected to solve some open questions related to the quark structure and confinement. Nambu proposed a generalisation of Hamiltonian formalism of mechanics based on the Liouville theorem of classical mechanics which is related to the invariance of the phase space volume under canonical transformations [11]. However, first attempts to understand the kind of mechanics involved within such formalism were not fruitful leaving the question to the exploration of some similarities and interrelations between Hamiltonian formalism and Newton mechanics. It is only by considering Yamaleev's construction that we obtain a complete realisation of Nambu formalism into real mechanics $[16,17,18]$. 
The main characteristic of this interpretation is factorisation, which is well known to us from spinor treatments or even from twistorial approaches [8], to mention some examples. Experience shows us that spinor and twistorial treatments lead to unclear results that are difficult to apply and interpret physically. However, Yamaleev's dynamics framework allows us to furnish factorisation with a physically meaningful interpretation.

Using the Yamaleev's approach, it is straightforward to find out the kind of solutions corresponding to the relativistic oscillator model. We even pursue to show the parallelism between this system and the mechanical properties and well known behaviour of the asymmetric top from the rigid body theory [9]. As is well known, the notion of a rigid body is in contradiction with the statement of an upper bound for the velocities. In this work we explore a plausible mapping between the dynamical solution of the relativistic oscillator, obtained within the mentioned formalism, and the one related to Euler's free rigid body. Such mapping will be permissible due to the fact that both systems possess solutions in terms of the Jacobian elliptic functions. As we will see, it is necessary to impose some restrictions in order to avoid any contradiction with relativistic mechanics.

We are not pretending to present the complete formal extension of the Nambu formalism, but the reader is referred to the literature for reviews on the topic. Some examples of other real physical systems described by Nambu's formalism (as well as proposals for quantised versions of Nambu dynamics) can be found in $[2,3,4,6,7,10,11,12,13,14,15]$. We start by briefly reviewing the main ideas within Nambu formalism.

Let $(x, y, z)=: \vec{r}$ be a dynamical triplet of variables that generates a three dimensional phase space. This space could be seen as a generalisation of the conventional two dimensional phase space with coordinates $(p, q)$. Let $H$ and $G$ be two functions of $(x, y, z)$ that will serve as a pair of Hamiltonian-like functions from which we want to obtain the dynamical evolution of the points in the triplet phase space. Nambu equations are postulated as

$$
\begin{aligned}
& \frac{d x}{d t}=\frac{\partial(H, G)}{\partial(y, z)}, \\
& \frac{d y}{d t}=\frac{\partial(H, G)}{\partial(z, x)}, \\
& \frac{d z}{d t}=\frac{\partial(H, G)}{\partial(x, y)},
\end{aligned}
$$

or in vector notation,

$$
\frac{d \vec{r}}{d t}=\vec{\nabla} H \times \vec{\nabla} G
$$

Hence, for any function $F=F(x, y, z)$ we will have

$$
\frac{d F}{d t}=\frac{\partial(F, H, G)}{\partial(x, y, z)}=\vec{\nabla} F \cdot(\vec{\nabla} H \times \vec{\nabla} G) .
$$

Next, we will define for arbitrary functions $F_{1}, F_{2}, F_{3}$ a generalised Poisson bracket $\left\{F_{1}, F_{2}, F_{3}\right\}$ by

$$
\left\{F_{1}, F_{2}, F_{3}\right\}=\vec{\nabla} F_{1} \cdot\left(\vec{\nabla} F_{2} \times \vec{\nabla} F_{3}\right) .
$$


This generalised Poisson bracket is antisymmetric under interchange of any pair of functions. From (1.3) and the antisymmetric property of (1.4) follows that both $H$ and $G$ are constants of motion.

In the same spirit, we can consider phase spaces of any finite dimension by introducing a vector $\vec{r}$ with $n$-components and $(n-1)$-Hamiltonian-like functions $H_{k}, k=0, \ldots,(n-1)$. In analogy to equations (1.2) and (1.3), we generalise the Nambu equations of motion

$$
\begin{aligned}
\frac{d x_{i}}{d t} & =\sum_{j, k, \cdots, l}^{n} \epsilon_{i j k \cdots l} \frac{\partial H_{1}}{\partial x_{j}} \frac{\partial H_{2}}{\partial x_{k}} \cdots \frac{\partial H_{n-1}}{\partial x_{l}}, \\
\frac{d F}{d t} & =\frac{\partial\left(F, H_{1}, H_{2}, \ldots, H_{n-1}\right)}{\partial\left(x_{1}, x_{2}, \ldots, x_{n-1}, x_{n}\right)}
\end{aligned}
$$

where $\epsilon_{i j k \cdots l}$ is the totally antisymmetric Levi-Civita tensor. ¿From this last generalisation it can be seen that the Hamiltonian formalism is contained in Nambu's formalism as a special case, namely, a two-dimensional phase space and a single Hamiltonian function. For further relations between Nambu and Hamiltonian formalisms, the reader is referred to $[3,7,10]$.

The rest of the paper is as follows. In section 2 we review Yamaleev's construction and interpretation of Nambu formalism of mechanics, and within this general framework we specialise to the relativistic harmonic oscillator case. In section 3 we recapitulate Euler's free asymmetric rigid body. The dynamical mapping between the solutions of the asymmetric top and those obtained for the relativistic oscillator within Yamaleev's approach are studied in section 4 . We also discuss in section 4 some mechanical restrictions to the mapping that emerge in order to make it physically meaningful. Finally, in section 5 we present some concluding remarks.

\section{Relativistic Yamaleev's framework}

In this section we will consider a simplified Nambu system based on a three dimensional phase space coordinatised by the triplet $\{x, p, q\}$ within the following physical interpretation $[16,17,18]$ : we take the physical system as formed by a real (corporeal) particle of mass $m$ which is localised in a given point by the coordinates $(x, P)$ of two dimensional phase space, hence the position of the real particle is given by the coordinate $x$ while its momentum is given by the coordinate $P$. This system will be considered as a composed system, formed by two subparticles with momenta $p$ and $q$, and masses $m_{p}$ and $m_{q}$, respectively; this situation is directly generalised to three dimensional triplet configuration space $(\vec{x}, \vec{p}, \vec{q})$. For convenience, the light velocity constant $c$ will be explicitly kept throughout the equations.

Under these conditions we will have two Hamiltonians, one for each of the subparticles, and we assume that they are given by the classical expresions

$$
\begin{aligned}
& H_{p}:=\frac{p^{2}}{2 m_{p}}+V(x), \\
& H_{q}:=\frac{q^{2}}{2 m_{q}}+V(x) .
\end{aligned}
$$


From Nambu equations (1.1) we obtain the following set of equations that we will call from now on Yamaleev equations of motion

$$
\begin{aligned}
& \frac{d p}{d \tau}=-\frac{d V}{d x} \frac{q}{m_{q}}, \\
& \frac{d q}{d \tau}=-\frac{d V}{d x} \frac{p}{m_{p}}, \\
& \frac{d x}{d \tau}=+\frac{p q}{m_{p} m_{q}} .
\end{aligned}
$$

Here $\tau$ is an evolution paremeter, but in agreement with the next equation, it will turn out to be the evolution proper time of the system. We claim that the system of equations $(2.2)$ is equivalent to the relativistic equations of motion for the real particle in the $(x, P)$-phase space. To show this, we will proceed as follows. First, we consider that the momentum $P$ and the velocity $d x / d \tau$ of the real particle are related in the usual way by the expression

$$
\frac{d x}{d \tau}=\frac{P}{m} .
$$

This relation must contain the same information as equation (2.2c). By comparison then we get that the momentum $P$ is factorised as a product of the individual momenta of the subparticles

$$
\begin{aligned}
P & :=\frac{p q}{2 \sqrt{m_{p} m_{q}}}, \\
m & :=\frac{\sqrt{m_{p} m_{q}}}{2},
\end{aligned}
$$

where $c$ stands for the speed of light. It is easy to show that we have two constants of motion given by $E_{p}:=H_{p}$ and $E_{q}:=H_{q}$, since we have

$$
\frac{d}{d \tau} E_{p}=\frac{d}{d \tau}\left(\frac{p^{2}}{2 m_{p}}+V(x)\right)=\frac{p}{m_{p}} \frac{d p}{d \tau}+\frac{d V}{d x} \frac{d x}{d \tau}=-\frac{d V}{d x}\left(\frac{p q}{m_{p} m_{q}}-\frac{d x}{d \tau}\right)=0,
$$

where we have used equations $(2.2 a, c)$ to obtain the last equality. An analogous proof follows for $E_{q}$.

Next, we will define two useful quantities in terms of these constants of motion. The total energy of the system $E$ will be considered as the arithmetic average, that is,

$$
E:=\frac{1}{2}\left(E_{p}+E_{q}\right),
$$

and we will identify the difference in energies as the rest mass of the system $\mathcal{M}$ as

$$
\mathcal{M} c^{2}:=\frac{1}{2}\left(E_{q}-E_{p}\right) .
$$

It is important to distinguish the quantity $\mathcal{M}$ (which is a constant of motion) from the parameter $m$ that appears in the dynamical equation (2.3). In terms of these two quantities the constants of motion $E_{p}$ and $E_{q}$ read

$$
\begin{aligned}
& E_{p}=E-\mathcal{M} c^{2}, \\
& E_{q}=E+\mathcal{M} c^{2} .
\end{aligned}
$$


Note that a third constant of motion can be obtained if we multiply $E_{p}$ and $E_{q}$

$$
\begin{aligned}
E_{p} E_{q} & =\left(E-\mathcal{M} c^{2}\right)\left(E+\mathcal{M} c^{2}\right)=E^{2}-\left(\mathcal{M} c^{2}\right)^{2}=\left(\frac{p^{2}}{2 m_{p}}+V(x)\right)\left(\frac{q^{2}}{2 m_{q}}+V(x)\right) \\
& =P^{2} c^{2}+2(E-V) V+V^{2} .
\end{aligned}
$$

From the third and last equality terms of (2.8) it follows that

$$
(E-V)^{2}=P^{2} c^{2}+\left(\mathcal{M} c^{2}\right)^{2},
$$

which is equivalent to

$$
E=\sqrt{P^{2} c^{2}+\left(\mathcal{M} c^{2}\right)^{2}}+V(x),
$$

which is the famous relation for the relativistic energy. Therefore, we reach Yamaleev's conclusion that two subparticles in Nambu's formalism are equivalent to an inertial (corporeal) relativistic particle. Furthermore, Yamaleev's approach allows to consider zero mass particles, due to the emerging distinction between the rest mass $\mathcal{M}$ and the dynamical parameter $m$. This is very important to point out because it is normally believed that zero mass particles are totally from a quantum mechanical nature.

A remarkable feature of Nambu's formalism is that the system of equations (2.2) satisfies a set of canonical equations of motion. In order to show this we consider the derivative of the product of the momenta of the two subparticles, that is,

$$
\frac{d}{d \tau}(p q)=-2 \frac{d V}{d x}\left(\frac{p^{2}}{2 m_{p}}+\frac{q^{2}}{2 m_{q}}\right)=-2 \frac{d}{d x}\left(2 E V-V^{2}\right) .
$$

In terms of the corporeal particle momenta $P$ this relation reads

$$
\frac{d}{d \tau} P=\frac{-1}{2 \mathcal{M} c^{2}} \frac{d}{d x}\left(2 E V-V^{2}\right)=-\frac{d W(x)}{d x},
$$

where we have identified the rest mass $\mathcal{M}$ with the dynamical parameter $m$, and we defined the potential function $W(x):=\left[2 E V(x)-V^{2}(x)\right] / 2 \mathcal{M} c^{2}$. Therefore, from equations (2.3) and (2.12), we obtain the Hamiltonian equations of motion

$$
\begin{aligned}
& \frac{d x}{d \tau}=\frac{\partial \mathcal{H}}{\partial P}, \\
& \frac{d P}{d \tau}=-\frac{\partial \mathcal{H}}{\partial x},
\end{aligned}
$$

where $\mathcal{H}:=\left(P^{2} / 2 \mathcal{M}\right)+W(x)$ is the Hamiltonian of the corporeal particle. Therefore, a relativistic particle under the potential field $V(x)$ is equivalent to a classical particle under the potential field $W(x)$.

In a similar fashion, the set of equations (2.2) can be expressed for three dimensional space as

$$
\begin{aligned}
& \frac{d \vec{p}}{d \tau}=-\vec{\nabla} V \times \frac{\vec{q}}{m_{q} c}, \\
& \frac{d \vec{q}}{d \tau}=-\vec{\nabla} V \times \frac{\vec{p}}{m_{p} c}, \\
& \frac{d \vec{x}}{d \tau}=+\frac{\vec{p} \times \vec{q}}{m_{p} m_{q}} .
\end{aligned}
$$


It is important to realize that the structure of Lorentz's force law in space time $\frac{d}{d \tau} P_{\mu}=$ $F_{\mu}{ }^{\nu} P_{\nu}$ corresponds to an evolution equation; it is also interesting to find out that, in the same way, the equations of motion above for the mechanical system of a relativistic particle also correspond to the structure of an evolution equation.

\subsection{Harmonic oscillator potential}

Now, we specialise the above developments to the unidimensional harmonic oscillator case, and hence, we will consider the potential $V(x)=m \omega^{2} x^{2} / 2$. We will now see, how easy it is to find, in the formalism, a solution to the harmonic relativistic oscillator. First, we consider the momenta for the corporeal particle given in equation (2.3), and we factorise this momenta as in equation (2.4a), $P=p q / 4 m c^{2}$, where $p$ and $q$ can be written in terms of the constants of motion $E_{p}$ and $E_{q}$ by means of the relations (2.1). This factorisation leads us to the integral (see reference [3] for further details)

$$
\tau-\tau_{0}=\int_{x\left(\tau_{0}\right)}^{x(\tau)} \frac{m c d x}{\sqrt{\left(E-\mathcal{M} c^{2}-V(x)\right)\left(E+\mathcal{M} c^{2}-V(x)\right)}}
$$

which for the one-dimensional oscillator potential $V(x)=m \omega^{2} x^{2} / 2$ reduces to the elliptic integral

$$
\phi=\int_{y\left(\phi_{0}\right)}^{y(\phi)} \frac{d y}{\sqrt{\left(1-y^{2}\right)\left(1-\kappa y^{2}\right)}},
$$

where we made the change of variable

$$
y:=\sqrt{\frac{m \omega^{2}}{2\left(E-\mathcal{M} c^{2}\right)}} x,
$$

and we defined

$$
\begin{aligned}
\phi & :=\omega\left(\tau-\tau_{0}\right) \sqrt{\frac{\left(E+\mathcal{M} c^{2}\right)}{2 m}}, \\
\kappa & :=\frac{E-\mathcal{M} c^{2}}{E+\mathcal{M} c^{2}} .
\end{aligned}
$$

As $0<\kappa<1$, solutions to integral (2.16) can be found by means of Jacobian elliptic functions $\operatorname{sn}(\phi, \kappa), c n(\phi, \kappa)$ and $d n(\phi, \kappa)[1,5]$. Jacobian elliptic functions obey similar algebraic identities to the trigonometric functions, namely

$$
\begin{aligned}
& c n^{2}(\phi \mid \kappa)+s n^{2}(\phi \mid \kappa)=1, \\
& d n^{2}(\phi \mid \kappa)+\kappa s n^{2}(\phi \mid \kappa)=1,
\end{aligned}
$$

and besides, they satisfy the differential identities

$$
\begin{aligned}
& \frac{d}{d \phi} c n(\phi \mid \kappa)=-s n(\phi \mid \kappa) d n(\phi \mid \kappa), \\
& \frac{d}{d \phi} \operatorname{sn}(\phi \mid \kappa)=d n(\phi \mid \kappa) c n(\phi \mid \kappa), \\
& \frac{d}{d \phi} d n(\phi \mid \kappa)=-\kappa \operatorname{sn}(\phi \mid \kappa) c n(\phi \mid \kappa) .
\end{aligned}
$$


Furthermore, in the limit $\kappa \rightarrow 0$ the Jacobian elliptic functions behave as $\operatorname{sn}(\phi \mid \kappa) \rightarrow$ $\sin (\phi), \operatorname{cn}(\phi \mid \kappa) \rightarrow \cos (\phi)$, and $d n(\phi \mid \kappa) \rightarrow 1$, respectively.

Differential identities (2.19) are equivalent to the set of equations (2.2) when considering the potential $V=m \omega^{2} x^{2} / 2$ and by taking the correspondence

$$
\begin{aligned}
x & :=\sqrt{\frac{2\left(E-\mathcal{M} c^{2}\right)}{m \omega^{2}}} \operatorname{sn}(\phi \mid \kappa), \\
p & :=\sqrt{2\left(E-\mathcal{M} c^{2}\right) m_{p}} \operatorname{cn}(\phi \mid \kappa), \\
q & :=\sqrt{2\left(E+\mathcal{M} c^{2}\right) m_{q}} d n(\phi \mid \kappa),
\end{aligned}
$$

or, in terms of the corporeal particle momenta,

$$
\begin{aligned}
& x=\sqrt{\frac{2\left(E-\mathcal{M} c^{2}\right)}{m \omega^{2}}} \operatorname{sn}(\phi \mid \kappa), \\
& P=\frac{\sqrt{E^{2}-\left(\mathcal{M} c^{2}\right)^{2}}}{c} \operatorname{cn}(\phi \mid \kappa) d n(\phi \mid \kappa) .
\end{aligned}
$$

Solutions (2.21) reduce to the non-relativistic oscillator in the limits $\mathcal{M}=m$ and $E_{n r} \ll$ $m c^{2}$, where $E_{n r}$ is the non-relativistic energy given by the expression $E=m c^{2}+E_{n r}$. These limits correspond to taking the limit $\kappa \rightarrow 0$ in Jacobian elliptic functions, that are reduced to the sine-cosine functions. Then, we obtain in such limit the solutions

$$
\begin{aligned}
x & =\sqrt{\frac{2 E_{n r}}{m \omega^{2}}} \sin \phi, \\
P & =\sqrt{2 m E_{n r}} \cos \phi, \\
\phi & =\omega\left(\tau-\tau_{0}\right),
\end{aligned}
$$

which are identified with the non-relativistic harmonic oscillator solutions for the corporeal point particle.

As we will see in next section, Euler's rigid body can also be cast in terms of Jacobian elliptic functions. This will give a point of comparison for the relativistic oscillator and the rigid body.

\section{Euler's rigid body}

In this section we briefly review free motion of the asymmetric rigid body in terms of Jacobian elliptic functions described above [9]. Let $\vec{\Omega}=\left(\Omega_{1}, \Omega_{2}, \Omega_{3}\right)$ be the angular velocity of the rigid body, and $\left(I_{1}, I_{2}, I_{3}\right)$ the principal momenta of inertia. As we will consider an asymmetrical rigid body, we take $I_{3} \neq I_{2} \neq I_{1}$, and without loss of generality we will work on the assumption that $I_{3}>I_{2}>I_{1}>0$. 
Free motion of the asymmetric rigid body is then described by Euler equations

$$
\begin{aligned}
& \frac{d \Omega_{1}}{d t}+\left(\frac{I_{3}-I_{2}}{I_{1}}\right) \Omega_{2} \Omega_{3}=0, \\
& \frac{d \Omega_{2}}{d t}+\left(\frac{I_{1}-I_{3}}{I_{2}}\right) \Omega_{3} \Omega_{1}=0, \\
& \frac{d \Omega_{3}}{d t}+\left(\frac{I_{2}-I_{1}}{I_{3}}\right) \Omega_{1} \Omega_{2}=0 .
\end{aligned}
$$

As is well known, the dynamics of the rigid body, described by these equations, possesses two integrals of motion:

$$
\begin{aligned}
\mathcal{E} & :=\frac{1}{2}\left(I_{1} \Omega_{1}^{2}+I_{2} \Omega_{2}^{2}+I_{3} \Omega_{3}^{2}\right), \\
J^{2} & :=I_{1}^{2} \Omega_{1}^{2}+I_{2}^{2} \Omega_{2}^{2}+I_{3}^{2} \Omega_{3}^{2},
\end{aligned}
$$

where $\mathcal{E}$ is the kinetic energy and $J:=\|\vec{J}\|$ is the total angular momentum of the free rigid body. As it was first realised by Nambu [11], Euler equations for a three dimensional rigid body can be cast within the formalism of Nambu mechanics (1.1) if we identify $\vec{r}=(x, y, z)$ with the components of the total angular momentum vector $\vec{J}$, and we take the pair of Hamiltonian-like functions, $H_{1}$ and $H_{2}$, as the total kinetic energy $\mathcal{E}(3.2 \mathrm{a})$, and the total angular momentum squared $J^{2}(3.2 \mathrm{~b})$, respectively.

Following [6], we introduce the new variable

$$
\psi:=\sqrt{\frac{\left(I_{3}-I_{2}\right)\left(J^{2}-2 \mathcal{E} I_{1}\right)}{I_{1} I_{2} I_{3}}} t,
$$

and the parameter $k$ by

$$
k:=\frac{\left(I_{2}-I_{1}\right)\left(2 \mathcal{E} I_{3}-J^{2}\right)}{\left(I_{3}-I_{2}\right)\left(J^{2}-2 \mathcal{E} I_{1}\right)} .
$$

Bearing on mind definitions (3.3) it is straightforward to show that the solutions of equations (3.1) can also be represented by Jacobian elliptic functions if we take

$$
\begin{aligned}
& \Omega_{1}=\sqrt{\frac{2 \mathcal{E} I_{3}-J^{2}}{I_{1}\left(I_{3}-I_{1}\right)}} \operatorname{cn}(\psi \mid k), \\
& \Omega_{2}=\sqrt{\frac{2 \mathcal{E} I_{3}-J^{2}}{I_{2}\left(I_{3}-I_{2}\right)}} \operatorname{sn}(\psi \mid k), \\
& \Omega_{3}=\sqrt{\frac{J^{2}-2 \mathcal{E} I_{1}}{I_{3}\left(I_{3}-I_{1}\right)}} d n(\psi \mid k) .
\end{aligned}
$$

Note that all the coefficients appearing in equations (3.3) and (3.4) are positive due to our original convention $I_{3}>I_{2}>I_{1}>0$. In particular, it follows that the parameter $k$ in $(3.3 \mathrm{~b})$ is defined within the interval $0<k<1$. Therefore, by direct substitution of (3.4) into equations (3.1) we recover the system of equations satisfied by the Jacobian elliptic functions (2.19).

Once we have realised that both systems possess their solutions in terms of the Jacobian elliptic functions that we come to the idea to establish a correspondence between the relativistic oscillator model and the free rigid body model. 


\section{Dynamical mapping}

In order to put in correspondence the solutions (2.20) and (3.4) we shall present the variables in (2.20) as variables with the dimensionality of frequency. For that purpose we shall use the auxiliary parameter $\ell$ with dimensionality of time which can be defined as $\ell=\omega^{-1}$. On making use of this parameters we rewrite (2.20) as follows

$$
\begin{aligned}
& \frac{x}{\ell^{2}}=\omega \sqrt{\frac{2 E_{p}}{m}} \operatorname{sn}(\phi \mid \kappa), \\
& \frac{p}{2 m \ell}=\omega \sqrt{\frac{2 E_{p}}{m_{q}}} \operatorname{cn}(\phi \mid \kappa), \\
& \frac{q}{2 m \ell}=\omega \sqrt{\frac{2 E_{q}}{m_{p}}} \operatorname{dn}(\phi \mid \kappa) .
\end{aligned}
$$

Thus, we have two systems which solutions are given by Jacobian elliptic functions: on one side, the free rigid body equations depend on the momenta of inertia $I_{1}, I_{2}, I_{3}$; on the other side, the equations of the relativistic oscillator depend on the mass parameters $m_{q}, m_{p}$. Furthermore, in both systems we have two constants of motion and a single evolution parameter, and hence we have the sets of quantities $\left(\mathcal{E}, J^{2}, t\right)$ in the former, and $\left(E_{p}, E_{q}, \tau\right)$ in the latter. We want to explore a mapping between the two systems. As we will see in subsection 4.1 below, some restrictions on the mapping necessarily emerge to keep the mapping physically consistent.

First of all let us put in correspondence the parameter $\kappa$ defined in $(2.17 \mathrm{c})$ with the parameter $k$ defined in $(3.3 \mathrm{~b})$. Comparing these formulae we define

$$
\begin{aligned}
& E_{p}:=\frac{1}{2}\left(\frac{2 \mathcal{E} I_{3}-J^{2}}{I_{3}-I_{2}}\right), \\
& E_{q}:=\frac{1}{2}\left(\frac{J^{2}-2 \mathcal{E} I_{1}}{I_{2}-I_{1}}\right) .
\end{aligned}
$$

Now, we proceed to compare the functions given by (4.1a) and (3.4b). These two functions will be equal if we take

$$
\frac{2 E_{p} \omega^{2}}{m}=\frac{2 \mathcal{E} I_{3}-J^{2}}{I_{2}\left(I_{3}-I_{2}\right)} .
$$

Taking into account mapping (4.2), hence we get the relation

$$
\frac{m}{\omega^{2}}=I_{2} \text {. }
$$

Analogously, we compare (4.1b) with (3.4a) and (4.1c) with (3.4c). These pairs of functions will be correspondingly equal, if we take

$$
\begin{aligned}
& \frac{2 E_{p} \omega^{2}}{m_{q}}=\frac{2 \mathcal{E} I_{3}-J^{2}}{I_{1}\left(I_{3}-I_{1}\right)}, \\
& \frac{2 E_{q} \omega^{2}}{m_{p}}=\frac{J^{2}-2 \mathcal{E} I_{1}}{I_{3}\left(I_{3}-I_{1}\right)},
\end{aligned}
$$


and again by using definitions (4.2), we find

$$
\begin{aligned}
& \frac{m_{q}}{\omega^{2}}=\frac{I_{1}\left(I_{3}-I_{1}\right)}{I_{3}-I_{2}}, \\
& \frac{m_{p}}{\omega^{2}}=\frac{I_{3}\left(I_{3}-I_{1}\right)}{I_{2}-I_{1}} .
\end{aligned}
$$

¿From relations (4.6) we obtain the following important identities

$$
\begin{aligned}
\omega^{2}\left(\frac{I_{1}}{m_{q}}+\frac{I_{3}}{m_{p}}\right) & =1, \\
\omega^{2}\left(\frac{1}{m_{p}}+\frac{1}{m_{q}}\right) & =\frac{I_{3}+I_{1}-I_{2}}{I_{1} I_{3}},
\end{aligned}
$$

which will serve (together with equations (4.6)) to obtain the further relation

$$
I_{3}-I_{1}=\frac{1}{\omega^{2}} \sqrt{m\left(m_{p}+m_{q}\right)-m_{p} m_{q}}=\frac{M_{p q}}{\omega^{2}},
$$

where we have defined $M_{p q}:=\sqrt{m\left(m_{p}+m_{q}\right)-m_{p} m_{q}}=\sqrt{m}\left(\left|\sqrt{m_{p}}-\sqrt{m_{q}}\right|\right)$. We see that $M_{p q}$ must be grater than zero due to $I_{3}-I_{1}>0$ and hence we assume for definiteness $m_{p}>m_{q}$ from now on. Relation (4.8) together with the identity (4.7a) can be solved for $I_{1}$ and $I_{3}$ in terms of the masses of the subparticles $m_{p}$ and $m_{q}$ by

$$
\begin{aligned}
& I_{1}=\frac{1}{\omega^{2}\left(m_{p}+m_{q}\right)}\left(m_{p} m_{q}-m_{q} M_{q p}\right), \\
& I_{3}=\frac{1}{\omega^{2}\left(m_{p}+m_{q}\right)}\left(m_{p} m_{q}+m_{p} M_{q p}\right) .
\end{aligned}
$$

Next, by direct algebraic calculations it can be shown that the constants of motion of the rigid body, $\mathcal{E}$ and $J^{2}$, can be written in terms of the relativistic oscillator parameters by

$$
\begin{aligned}
\mathcal{E}= & \frac{\left(m_{p}-M_{p q}\right) E_{p}-\left(m_{q}+M_{p q}\right) E_{q}}{m_{p}+m_{q}}, \\
J^{2}= & {\left[-\left(m_{q}+M_{p q}\right)\left(m_{p} m_{q}-m_{p} M_{p q}-2 m_{q} M_{p q}\right) E_{q}+\left(m_{p}-M_{p q}\right)^{2} m_{q} E_{p}\right] } \\
& \times\left[\frac{1}{\omega^{2}\left(m_{p}+m_{q}\right)^{2}}\right] .
\end{aligned}
$$

Finally, let us compare the two expressions for the evolution parameter $\phi$ and $\psi$, where the former was defined in (2.17b) and the latter, in (3.3a). On equating these two definitions we obtain

$$
\begin{aligned}
\tau-\tau_{0} & =t\left[\left(\frac{\left(I_{3}-I_{2}\right)\left(J^{2}-2 \mathcal{E} I_{1}\right)}{I_{1} I_{2} I_{3}}\right)\left(\frac{2 m}{E_{q} \omega^{2}}\right)\right]^{\frac{1}{2}}=t\left[\left(\frac{\left(I_{3}-I_{2}\right)\left(I_{2}-I_{1}\right)}{I_{1} I_{2} I_{3}}\right)\left(\frac{4 m}{\omega^{2}}\right)\right]^{\frac{1}{2}} \\
& =2 t\left[\frac{\left(I_{3}-I_{2}\right)\left(I_{2}-I_{1}\right)}{I_{1} I_{3}}\right]^{\frac{1}{2}}
\end{aligned}
$$


Table 1: Relativistic oscillator parameters in terms of Euler's rigid body parameters

$$
\begin{aligned}
\frac{m}{\omega^{2}} & =I_{2} \\
\frac{m_{p}}{\omega^{2}} & =\frac{I_{3}\left(I_{3}-I_{1}\right)}{I_{2}-I_{1}} \\
\frac{m_{q}}{\omega^{2}} & =\frac{I_{1}\left(I_{3}-I_{1}\right)}{I_{3}-I_{2}} \\
E_{p} & =\frac{1}{2}\left(\frac{2 \mathcal{E} I_{3}-J^{2}}{I_{3}-I_{2}}\right) \\
E_{q} & =\frac{1}{2}\left(\frac{J^{2}-2 \mathcal{E} I_{1}}{I_{2}-I_{1}}\right) \\
\tau-\tau_{0} & =\frac{\left(I_{3}-I_{1}\right)}{I_{2}} t
\end{aligned}
$$

where we used relations (4.2) and (4.4) to obtain the second and third equalities, respectively. This relation can be easily inversed to put the parameter $t$ completely in terms of relativistic oscillator parameters by means of relations (4.6) and (4.8)

$$
t=\frac{1}{\sqrt{2}\left(\frac{m_{p}+m_{q}}{\sqrt{m_{p} m_{q}}}-2\right)^{\frac{1}{2}}}\left(\tau-\tau_{0}\right)=\frac{\sqrt{m}}{\left|\sqrt{m_{p}}-\sqrt{m_{q}}\right|}\left(\tau-\tau_{0}\right) .
$$

Thus, so far we have obtained a general algebraic well defined mapping between the two systems. The maps from one system to the other are summarised in tables 1 and 2 . The only remaining question is to explore the restrictions that we have to consider in order to obtain a physically meaningful mapping between the two systems.

\subsection{Restrictions to the mapping}

In this subsection we will describe the emerging restrictions that the mapping described above should have in order to correspond to the physical systems studied. Such restrictions are related to the definition of the corporeal particle mass $m$ in terms of the masses of the subparticles $m_{p}$ and $m_{q}$, on one side; and to the conventions we considered for the principal momenta of inertia, on the other. However, a more important restriction will emerge in order to assure that the velocity of the corporeal particle remains upper bounded in agreement with the principles of special relativity.

To start, we consider the definition $(2.4 \mathrm{~b})$ of the corporeal mass $m$ in terms of the masses $m_{p}$ and $m_{q}$. By equations (4.4) and (4.6), this definition will hence imply that the principal momenta of inertia are not independent but forced to obey the algebraic relation

$$
4 I_{2}^{2}=\frac{I_{1} I_{3}\left(I_{3}-I_{1}\right)^{2}}{\left(I_{3}-I_{2}\right)\left(I_{2}-I_{1}\right)} .
$$


Table 2: Euler's rigid body parameters in terms of the relativistic harmonic oscillator parameters

$$
\begin{aligned}
I_{2} & =\frac{m}{\omega^{2}} \\
I_{1} & =\frac{m_{p} m_{q}-m_{q} M_{p q}}{\omega^{2}\left(m_{p}+m_{q}\right)} \\
I_{3} & =\frac{m_{p} m_{q}+m_{p} M_{p q}}{\omega^{2}\left(m_{p}+m_{q}\right)} \\
\mathcal{E} & =\frac{\left(m_{p}-M_{p q}\right) E_{p}-\left(m_{q}+M_{p q}\right) E_{q}}{m_{p}+m_{q}} \\
J^{2} & =\frac{\left\{\left(m_{p}-M_{p q}\right)^{2} m_{q} E_{p}-\left(m_{q}+M_{p q}\right)\left(m_{p} m_{q}-m_{p} M_{p q}-2 m_{q} M_{p q}\right) E_{q}\right\}}{\omega^{2}\left(m_{p}+m_{q}\right)^{2}} \\
t & =\frac{m}{M_{p q}}\left(\tau-\tau_{0}\right)
\end{aligned}
$$

Therefore the relativistic oscillator imposes a restriction on the way we have to choose the principal momenta of inertia. We can make use of this restriction to write the relations (4.11) and (4.12) between the evolution parameters in a simpler form, namely,

$$
\begin{aligned}
& \tau-\tau_{0}=\frac{I_{3}-I_{1}}{I_{2}} t, \\
& t=\frac{m}{M_{p q}}\left(\tau-\tau_{0}\right) .
\end{aligned}
$$

On the other side, we are working on the general convention that the principal momenta of inertia of the rigid body obeys the chain of inequalities $I_{3}>I_{2}>I_{1}>0$. Besides, as is well known, from the definitions of these momenta [9] we have also to consider the further inequalities $I_{i}+I_{j}>I_{k}$ for $i, j, k$ cyclic and where $i, j, k$ take values in $\{1,2,3\}$. ¿From equation (4.9a) we see that the inequality $I_{1}>0$ will only be satisfied if we take $m_{p}>m$. Similarly, by equations (4.4) and (4.8), the inequality $I_{1}+I_{2}>I_{3}$ will imply $m_{q}>m$. It is easy to show that the rest of the inequalities are automatically satisfied as a consequence of $m_{p}>m$ and $m_{q}>m$. Therefore the inequalities followed by the principal momenta of inertia restricts the choice of the masses of the subparticles $m_{p}$ and $m_{q}$. In particular, we can take these masses as $m_{p}=\alpha m$ and $m_{q}=\beta m$ where the constants $\alpha$ and $\beta$ are greater than one and $\alpha \beta=4$, in agreement with the definition (2.4b), and hence $\alpha$ and $\beta$ are restricted to take values in the intervals $1<\alpha<4$ and $1<\beta<4$.

Finally, we have to consider that the velocities of the corporeal particle with mass $m$ has an upper bound as required by the theory of special relativity. This implies that the velocity (2.3) is restricted to be less or equal to the speed of light, $c$, in absolute value. With this on mind, we substitute (2.21b) in the velocity (2.3), and since $|\operatorname{cn}(\phi \mid \kappa)| \leq 1$ and $|d n(\phi \mid \kappa)| \leq 1[1,5]$, we thus need to take $m c^{2} \geq \sqrt{E_{p} E_{q}}$ which in turn implies a condition 
Table 3: Restrictions to the mapping between the two systems

$$
\begin{aligned}
& m_{p}>m_{q}>m>0 \Longleftrightarrow \quad\left\{\begin{array}{l}
I_{3}>I_{2}>I_{1}>0 \\
I_{i}+I_{j}>I_{k} \quad(i, j, k \text { cyclic })
\end{array}\right. \\
& m=\frac{\sqrt{m_{p} m_{q}}}{2} \quad \Longleftrightarrow \quad 4 I_{2}^{2}=\frac{I_{1} I_{3}\left(I_{3}-I_{1}\right)^{2}}{\left(I_{3}-I_{2}\right)\left(I_{2}-I_{1}\right)} \\
& m c^{2} \geq \sqrt{E_{p} E_{q}} \quad \Longleftrightarrow \quad \omega^{4} \geq \frac{\left(2 \mathcal{E} I_{3}-J^{2}\right)\left(J^{2}-2 \mathcal{E} I_{1}\right)}{I_{1} I_{3}\left(I_{3}-I_{1}\right)^{2}}
\end{aligned}
$$

on the frequency $\omega$ of the harmonic oscillator given by

$$
\omega^{4} \geq \frac{1}{4} \frac{\left(2 \mathcal{E} I_{3}-J^{2}\right)\left(J^{2}-2 \mathcal{E} I_{1}\right)}{I_{2}^{2}\left(I_{3}-I_{2}\right)\left(I_{2}-I_{1}\right)}=\frac{\left(2 \mathcal{E} I_{3}-J^{2}\right)\left(J^{2}-2 \mathcal{E} I_{1}\right)}{I_{1} I_{3}\left(I_{3}-I_{1}\right)^{2}},
$$

where we used the restriction (4.13) to obtain the last inequality.

We claim that all these restrictions in the parameters of both the relativistic oscillator and the rigid body are physically well defined and consistent. The restrictions considered on this subsection are summarised in table 3 .

\section{Concluding remarks}

The purpose of the present work has been to show in an easy way, how Yamaleev's approach provides the bridge between the classical formalism of mechanics and the corresponding extension to the relativistic domain. In spite of the historical developments, it turns out not to be a quite extreme difficult task to provide solutions for the relativistic harmonic oscillator when we apply Yamaleev's formalism. The factorisation technique enable us to describe this relativistic system by means of its internal degrees of freedom. Furthermore, we have been able to discuss the remarkable parallelism between both solutions of the relativistic oscillator system and the corresponding mechanical properties of the Euler's free asymmetric rigid body. This turns out to be not just a mere formal mathematical curiosity, but this demonstrates that physically a relativistic oscillator like the asymmetric top is really a three dimensional system.

We also expect that a similar map for both systems can be expected at a quantum level, and we hope that our approach pave the way to a consistent quantum analysis of either system. This will be worked elsewhere.

Acknowledgments. AM acknowledges partial support from CONACYT (Mexico) under grant EP-050291.

\section{References}

[1] Abramowitz M and Stegun I A, Handbook of Mathematical functions, Dover Publications, New York, 1965. 
[2] Awata H, Li M, Minic D and Toneya T, On the quantization of Nambu brackets, JHEP 02 (2001), 013.

[3] Chatterjee R, Dynamical Symmetries and Nambu Mechanics, Lett. Math. Phys. 36 (1996), 117.

[4] CrÂşmăreanu M, First Integrals Generated by Pseudosymmetries in NambuPoisson Mechanics, J. Nonlin. Math. Phys. 7 (2000), 126-135.

[5] Gradshteyn I S and Ryzhik I M, Table of integrals, series and products, fourth edition, Academic, New York, 1980.

[6] Hirayama M, Realization of Nambu Mechanics: A particle interacting with an $S U(2)$ monopole, Phys. Rev. D16 (1977) 530-532.

[7] Homma T and Kadoya M, A relativistic particle with constraints in the Nambu mechanics, [KEK-preprint:9203637] (1991).

[8] Keller J, Rodríguez A and Yamaleev R M, An Algebraic Linear Relativistic Equation over Twistor Fields with Confined States, Adv. Appl. Clifford Algebras 6 (1996), 275.

[9] Landau L D and Lifshitz E M, Mechanics, Pergamon, London, 1959.

[10] Mukunda N and Sudarshan E C G, Relation between Nambu and Hamiltonian mechanics, Phys. Rev. D13 (1976), 2846-2850.

[11] Nambu Y, Generalized Hamiltonian Mechanics, Phys. Rev. D7 (1973), 2405-2412.

[12] Névir P and Blender R, A Nambu Representation of Incompressible Hydrodynamics using Helicity and Enstrophy, J. Phys. A 26 (1993), L1189-L1193.

[13] Pandit S A and Gangal A D, On Generalized Nambu Mechanics, J. Phys. A 31 (1998), 2899-2912.

[14] Razavy M and Kennedy F J, Generalized Phase Space Formulation of the Hamiltonian Dynamics, Can. J. Phys. 52 (1974).

[15] Takhtajan L, On Foundation of the Generalized Nambu Mechanics, Comm. Math. Phys. 160 (1994), 295.

[16] Yamaleev R M, Generalized Newtonian equations of motion, Ann. Phys. (NY) 277 (1999), 1-18.

[17] Yamaleev R M, Relativistic equations of motion within Nambu's formalism of dynamics, Ann. Phys. (NY) 285 (2000), 141-160.

[18] Yamaleev R M, Generalized Lorentz-Force equations, Ann. Phys. 292 (2001), 157178. 